treated surgically). Medical treatments were used in the majority (88.8\%) of TLSD cases. The most common medical treatments were non-steroidal anti-inflammatories $(n=782$, $74.2 \%)$, tramadol $(332,31.5 \%)$, gabapentin $(156,14.8 \%)$ and glucocorticoids (103, 9.8\%), with multimodal analgesia used in 401 (38.0\%) cases. The most popular complementary treatments were rest alone (45.8\%), rest and physiotherapy (2.7\%) and hydrotherapy (1.1\%). Overall, 113 (10.7\%) animals were euthanized as a result of their TLSD.

By utilizing large merged datasets from primary-care practices, a novel insight into caseload and management in the general UK dog population can be gained. The results of this study identified that the most common presenting breeds with TLSDs in the primary-care setting were crossbreds and Labrador Retrievers, although this is influenced by breed popularity and further risk factor analyses will be undertaken. The welfare impact of TLSDs was shown to be high, with most cases requiring long-term analgesia and often multimodal management. Furthermore one in ten TLSD cases were euthanized because of their condition.

\section{The occurrence of cardiac abnormalites in canine steroid responsive meningio-arteritis (SRMA)}

\section{Gerard McLauchlan', Jacques Penderis', Laura Dickson', Karen Holmes ${ }^{1}$, Jorge Prieto-Ramos', Laura Cosgrove', Rodrigo Gutierrez-Quintana', Julien Guevar', Inigo Sanz-Gonzalez', Anne French ${ }^{1}$}

\footnotetext{
1 University of Glasgow, Glasgow, UK

2 Vet Extra Neurology, Stirling, UK
}

Concurrent cardiac disorders, such as arrhythmias and myocarditis, are well-documented in human patients with central nervous system (CNS) diseases, including bacterial meningitis. Similarly, single case reports in dogs with steroid responsive meningio-arteritis (SRMA) have suggested concurrent cardiac abnormalities. This prospective study aimed to document the prevalence of cardiac changes in dogs with SRMA based on measurement of serum cardiac troponin-I (cTnl) and echocardiography, and to monitor these changes during SRMA treatment.

SRMA was diagnosed based on typical clinical presentation, blood/urinalysis, cerebral spinal fluid analysis, and in some cases infectious disease serology and MRI. Echocardiography was performed in all dogs, where possible prior to starting any anaesthesia, opiate or glucocorticoid therapy. Repeated neurological examination, C-reactive protein (CRP), cTnl and echocardiography were performed after 14 days.

Fourteen dogs were prospectively enrolled (3FE, 1FN, $5 \mathrm{ME}, 5 \mathrm{MN}$ ) with a mean age of 12.5 months (range 6 months - 30 months). Increased cTnl was identified in 5/14 dogs (mean $4.16 \mathrm{ng} / \mathrm{l}$; range 0.59-14.5; reference range $<0.15$ ) and echocardiographic abnormalities detected in $12 / 14$ dogs, including spontaneous echo-contrast (SEC) in 12/14 and mild pericardial effusion in 5/14

Treatment with glucocorticoids was associated with clinical improvement and CRP normalisation in all dogs. cTnl normalised in $4 / 5$ dogs and significantly reduced in $1 / 5$ dogs. SEC resolved in 9/12 dogs and reduced in $3 / 12$ dogs. Pericardial effusion resolved in all dogs.

These results show that cardiac changes are common in dogs with SRMA, and resolve with therapy in most dogs. Longer term follow-up of dogs with persistent cardiac changes is warranted. 